\title{
Retrospective Cohort Study of Venous Thromboembolism Rates in Ambulatory Cancer Patients: Association With Khorana Score and Other Risk Factors
}

\author{
Keziah Austina, d, Jessica George ${ }^{\text {a, d }}$, Emily J. Robinson ${ }^{\text {, }}$ \\ Marie Scully ${ }^{\mathrm{a}, \mathrm{c}}$, Mari R. Thomas ${ }^{\mathrm{a}, \mathrm{c}, \mathrm{e}}$
}

\begin{abstract}
Background: Guidelines do not recommend that cancer outpatients receive thromboprophylaxis unless at high venous thromboembolism (VTE) risk, with the Khorana score suggested for risk stratification. This study investigated VTE incidence in outpatients with pancreatic, endometrial, colorectal, ovarian and cervical cancer, the role of Khorana score in risk assessment and potential risk factors.
\end{abstract}

Methods: Data were retrospectively collected 1 year after cancer diagnosis. VTE associated with inpatient admissions was excluded.

Results: Seven hundred forty-six patients were included. VTE rates varied: $26.8 \%$ pancreatic; $5.7 \%$ endometrial; $9.8 \%$ colorectal; $10.2 \%$ ovarian; and $0.0 \%$ cervical cancer. Excluding VTE at diagnosis, potentially preventable VTE rates were $16.5 \%$ in pancreatic, $3.8 \%$ in endometrial, $9.8 \%$ in colorectal and $8.7 \%$ in ovarian cancer. Khorana score was associated with VTE in endometrial cancer only (high-risk: $16.7 \%$ vs. low-risk: $1.5 \%$; $\mathrm{P}<0.001)$. VTE rates for patients with central venous catheters (CVCs) were 22.6-34.8\% in pancreatic, endometrial, colorectal and ovarian cancers. VTE was associated with CVCs in endometrial, colorectal and ovarian; chemotherapy and $\mathrm{Hb}<100 \mathrm{~g} / \mathrm{L}$ in pancreatic; surgery in endometrial and ovarian; and body mass index $>$ 35 in ovarian cancers following adjusted analysis $(\mathrm{P}<0.05)$.

Conclusions: VTE is a significant burden in pancreatic, endometrial, colorectal and ovarian cancers. Khorana score was not predictive in most cancers. The major VTE-associated variable was CVC. Our data suggest a role for clinical trials of thromboprophylaxis in targeted

Manuscript submitted January 15, 2019, accepted March 20, 2019

aDepartment of Haematology, UCLH, 3rd Floor West, 250 Euston Road, London, NW1 2PG, UK

bDepartment of Biostatistics \& Health Informatics, Institute of Psychiatry, King's College London, De Crespigny Park, Denmark Hill, London, SE5 $8 \mathrm{AF}, \mathrm{UK}$

'Department of Haematology \& Cardiometabolic BRC, 1st Floor, Maple House, 149 Tottenham Court Road, London, W1T 7DN, UK

${ }^{\mathrm{d}}$ These authors were joint first authors.

${ }^{e}$ Corresponding Author: Mari R. Thomas, Department of Haematology, UCLH, 3rd Floor West, 250 Euston Road, London, NW1 2PG, UK.

Email: mari.thomas@nhs.net cancer outpatients.

Keywords: Venous thromboembolism; Khorana score; Ambulatory cancer; Thromboprophylaxis

\section{Introduction}

Venous thromboembolism (VTE) causes significant morbidity and mortality in patients with malignancy. The MEGA study (2005) demonstrated that the risk of VTE increased sevenfold in patients with cancer, and that the highest risk was immediately after diagnosis, in patients with metastatic disease, and in those with hematological malignancy [1]. Cancer VTE is the second leading cause of death in cancer patients, and it is a recognized independent risk factor for mortality [2]. Patients with cancer-associated thrombosis have an increased risk of VTE recurrence and major bleeding $[3,4]$.

Risk assessment for VTE is now routine for both medical and surgical inpatients in the UK. International guidelines recommend thromboprophylaxis in surgical inpatients with a moderate VTE risk of 3\%, provided bleeding risk is not high, and also in medical inpatients at a high risk $(11 \%)$ of VTE [5, 6]. However, the role of thromboprophylaxis in outpatients with cancer is less clear, with guidance recommending that prophylaxis is not used routinely, but considered for those at high thrombotic risk [5, 7]. Some guidelines suggest risk stratification using the Khorana score (Table 1) with thromboprophylaxis considered in patients with a high risk score $(\geq 3)[8,9]$.

We undertook a retrospective cohort study to investigate VTE incidence in the first year of diagnosis in outpatients diagnosed with pancreatic, endometrial, colorectal, ovarian or cervical cancer, at a large tertiary cancer center. We reviewed the association with Khorana score at cancer diagnosis and investigated the role of other risk factors in development of cancer VTE.

\section{Patients and Methods}

\section{Patient selection}

A retrospective identification of patients with a multidiscipli- 
Table 1. Khorana Score (Adapted From reference [9])

\begin{tabular}{|ll}
\hline Patient characteristics & Score \\
\hline Site of cancer & 2 \\
$\quad$ Very high risk (stomach and pancreas) & 1 \\
$\quad$ High risk (lung, lymphoma, gynecological, bladder and testicular) & 1 \\
Pre-chemotherapy platelet count $350 \times 10^{9}$ or more & 1 \\
Hemoglobin level less than $100 \mathrm{~g} / \mathrm{L}$ or use of red cell growth factors & 1 \\
Pre-chemotherapy leucocyte count more than $11 \times 10^{9} / \mathrm{L}$ & 1 \\
\hline BMI $35 \mathrm{~kg} / \mathrm{m}^{2}$ or more & 1 \\
\hline
\end{tabular}

BMI: body mass index.

nary team (MDT) diagnosis of pancreatic, endometrial, colorectal, ovarian or cervical cancer, between December 1, 2012 and December 31, 2014, or between December 1, 2009 and December 31, 2014 for pancreatic cancer patients (to ensure sufficient data) was conducted. Cases were excluded from analysis if: VTE occurred while an inpatient or within 90 days of discharge (NHS England, 2013); pre-existing anticoagulation at the time of VTE; patients who had incomplete data available to calculate a Khorana score; concurrent primary cancer or patients who did not receive all of their oncology care at the tertiary center and therefore with insufficient follow-up data. For the main analysis, patients with VTE diagnosed at or before cancer diagnosis were also excluded, as the aim was to calculate the role of the Khorana score in identifying patients at risk of a VTE which could have potentially been prevented with thromboprophylaxis (Fig. 1).

\section{Data collection}

For each patient, clinician reports (including clinic letters, chemotherapy planning documents, discharge summaries and MDT discussions), radiology reports, pathology results, electronic VTE risk assessments, chemotherapy regime and electronically stored patient demographics were reviewed. Information on the following variables was collected: patient age, ethnicity, body mass index (BMI), cancer type and stage, details of cancer therapy including chemotherapy, radiotherapy and surgical intervention, indwelling central venous catheters as well as details relating to any VTE events including date, site, clinical presentation and treatment. VTEs were recorded if documented by radiology report or clinician report of confirmed VTE event. Baseline/pre-chemotherapy blood tests were collated including hemoglobin level, leukocyte count, platelet count, C-reactive protein and creatinine level, and the Khorana score calculated. Cancer stage was divided into early and advanced for the purpose of analysis. Early stage was defined as attempt at curative resection in pancreatic cancer, FIGO stages 1-3 in colorectal cancer and FIGO stages 1-2 in both endometrial and ovarian cancer.

\section{Statistical analysis}

Descriptive statistics were reported for patient demograph- ics and treatment variables, stratified by cancer type to compare the different cohorts. For continuous variables statistics were reported in means (standard deviation (SD)) or medians (interquartile range (IQR)), where appropriate, and categorical variables were reported by $\mathrm{n}(\%)$. Clinical measures that are included in the Khorana score were grouped into high and

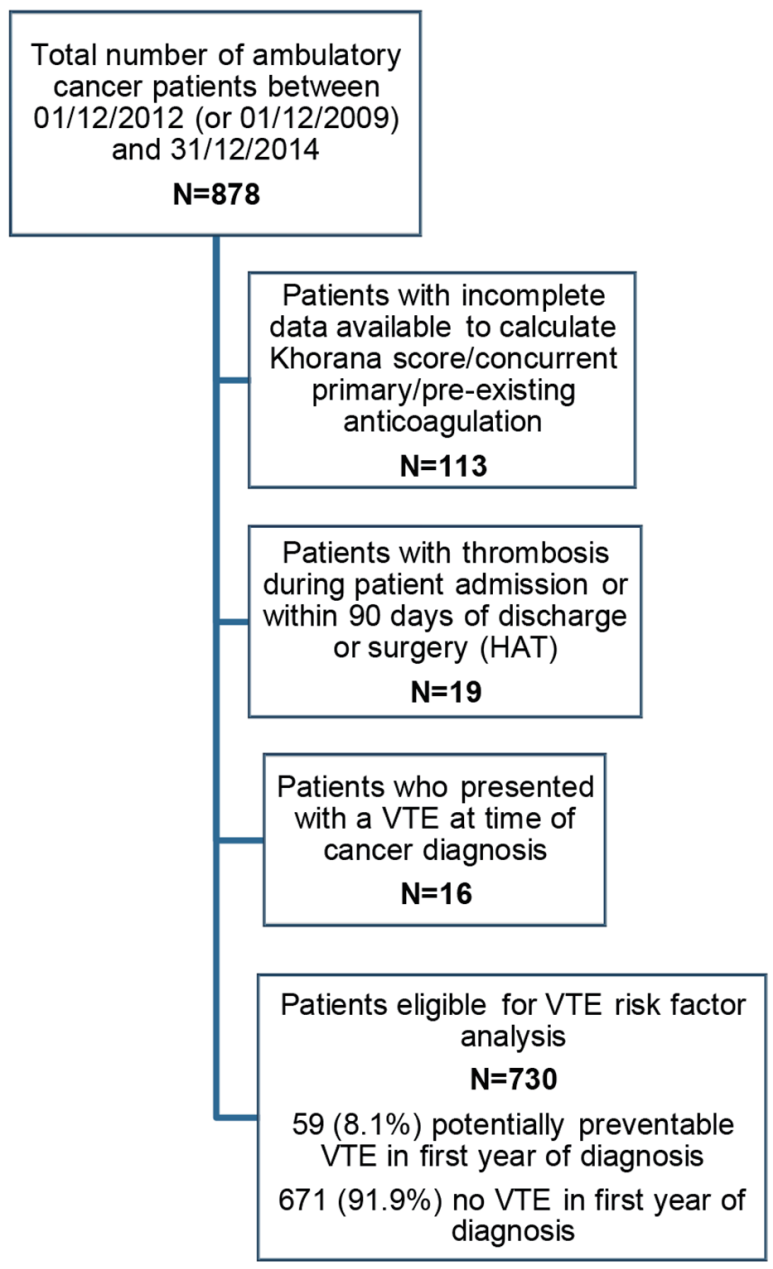

Figure 1. STROBE diagram to illustrate patient eligibility for the potentially preventable VTE risk factor analysis. VTE: venous thromboembolism. 
low risk categories, corresponding to the thresholds when calculating the score. Similarly, patients were split into low risk $($ Khorana score $<3$ ) vs. high risk (Khorana score $\geq 3$ ) for each cancer cohort. Abdominal VTE events were not included in the Khorana score analysis as such events were not included in the derivation of the score.

For the inferential analyses, a binary variable was created for VTE development (any vs. none). Univariate associations were tested between VTE development and clinical measures that were agreed a priori as potential risk factors of VTE using Pearson's Chi-squared test. Results were reported using the corresponding $\mathrm{P}$ values.

Stepwise logistic regression using backward elimination was then performed for each cancer type, using a significance level for removal from the model of $\alpha=0.05$. This tested which of the VTE risk factors remained statistically significant after adjusting for the other factors. All analysis was completed using Stata version 14.0 (StataCorp, College Station, TX, USA, 2015).

\section{Results}

There were 878 new diagnoses of pancreatic, endometrial, colorectal, ovarian and cervical cancer between December 1, 2012 (or December 1, 2009 for pancreatic) and December 31, 2014 (Fig. 1, STROBE diagram). One hundred thirteen patients were excluded from the study because of pre-existing anticoagulation or incomplete data. Nineteen further patients were excluded as the thrombotic event occurred during patient admission or within 90 days of discharge or surgery (hospital acquired thrombosis (HAT)). Seven hundred forty-six patients were therefore included in the study: 97 pancreatic cancer diagnoses, 157 endometrial cancer, 205 colorectal cancer, 196 ovarian cancer and 91 cervical cases. Patients who presented with a VTE at time of cancer diagnosis $(n=16)$ were excluded from the subsequent VTE risk factor analysis. Patient and treatment characteristics of patients included in the VTE risk factor analysis are shown in Table 2.

\section{VTE incidence}

Rates of total VTEs and potentially preventable VTEs (Fig. 2) were recorded for each cancer group.

\section{VTE demographics}

\section{Pancreatic cancer}

Twenty-six patients with VTE diagnosed during the first year of cancer diagnosis developed 31 individual clots (nine lower limb deep vein thrombosis (LL-DVT), three upper limb DVT (UL-DVT) (all central venous catheter (CVC)-associated), five pulmonary emboli (PE), one inferior vena cava (IVC) and 13 intra-abdominal thromboses comprising of portal, splenic, hepatic, superior mesenteric and renal vein thromboses). Of these patients, $13 / 26$ had symptomatic VTE, while the remaining patients had VTE incidentally found on staging scans; the majority of these were intra-abdominal VTE (five portal vein, four splenic vein, one hepatic vein, one renal vein and one superior mesenteric vein) as well as two incidental PEs. Of the patients, 14/26 were treated with low molecular weight heparin (LWMH). Untreated patients were asymptomatic or had contraindication to anticoagulation.

\section{Endometrial cancer}

Nine patients with VTE diagnosed during the first year of diagnosis developed 10 individual clots (two LL-DVT, one ULDVT (CVC-associated) and seven PE). Six out of nine were symptomatic, with three incidental PEs found on staging CT scans. All received treatment dose LMWH.

\section{Colorectal cancer}

Twenty patients with VTE diagnosed within the first year developed 21 individual VTE episodes (five LL-DVT, five ULDVT (all CVC-associated), eight PE, one neck and two portal vein thromboses). Nine out of 20 patients were asymptomatic (seven incidentally found PE and two incidentally found portal vein thromboses on staging scans). The majority of patients $(18 / 20)$ were treated with LMWH. Of the remaining two patients, one died prior to treatment commencing, and the other had a small non-occlusive CVC-associated DVT which was managed by removal of the line only.

\section{Ovarian cancer}

Twenty patients with VTE diagnosed within the first year developed 23 individual clots (five lower limb DVT, four upper limb DVT (all peripherally inserted central catheter (PICC)-associated), 12 pulmonary emboli and two inferior vena cava thromboses). Fourteen out of 21 patients had symptomatic VTE, seven asymptomatic, one patient had three individual events (one symptomatic PE, followed by separate lower limb and IVC thromboses incidentally found on staging scans). Twenty out of 21 patients received LMWH. In the remaining patient, anticoagulation was contraindicated because of high bleeding risk outweighing benefit for treating small incidental PEs.

\section{Cervical cancer}

None of the 91 patients diagnosed with cervical cancer had a VTE unrelated to an inpatient stay or surgery in the first year following diagnosis.

\section{Association with Khorana score}

Patients were divided into high risk $(\geq 3)$ and low risk $(<3)$ 
Table 2. Patient and Treatment Characteristics of Patients Included in the VTE Risk Factor Analysis

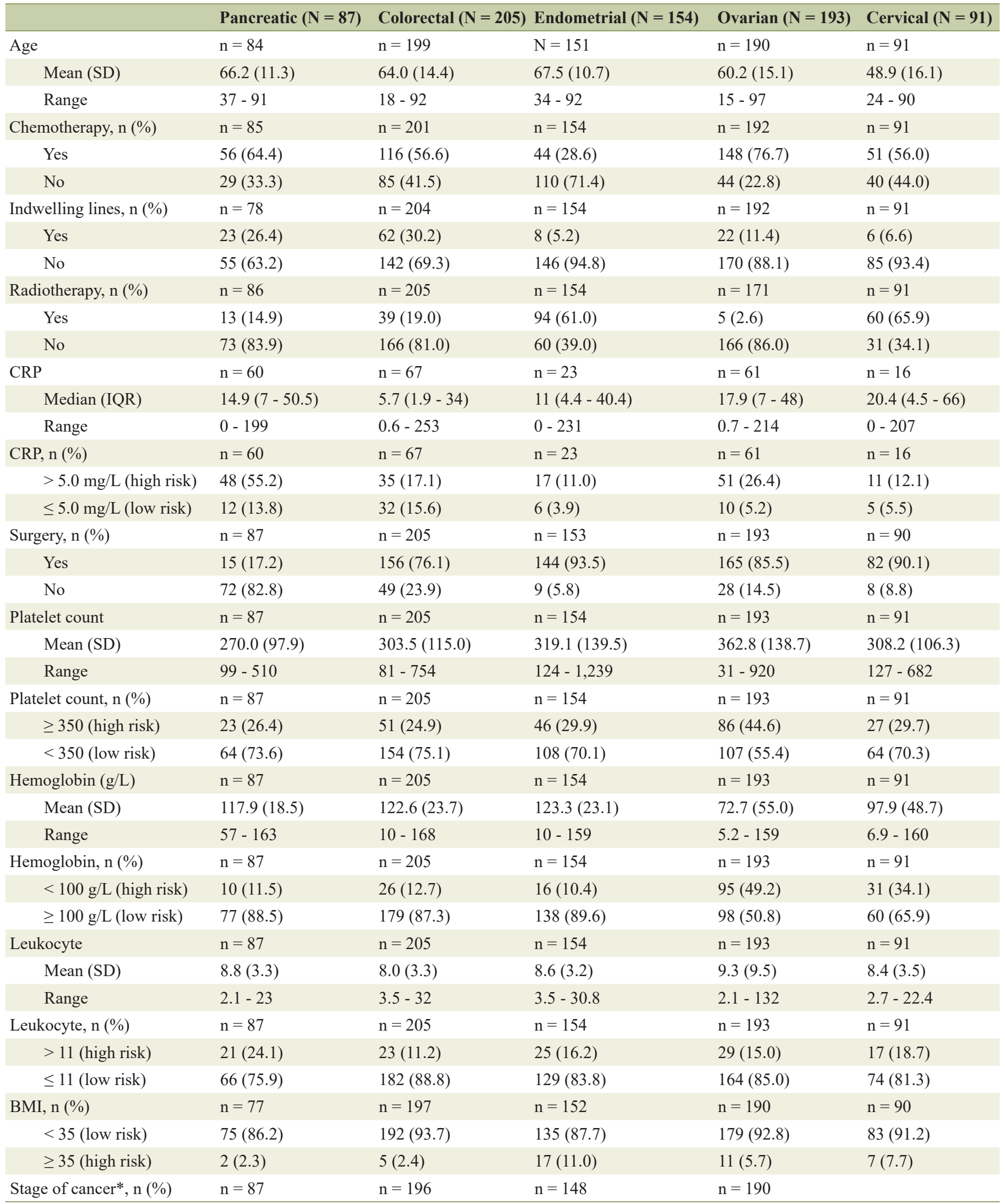


Table 2. Patient and Treatment Characteristics of Patients Included in the VTE Risk Factor Analysis - (continued)

\begin{tabular}{|c|c|c|c|c|c|}
\hline & Pancreatic $(\mathrm{N}=87)$ & Colorectal $(\mathrm{N}=\mathbf{2 0 5})$ & Endometrial $(\mathrm{N}=154)$ & Ovarian $(N=193)$ & Cervical $(\mathrm{N}=91)$ \\
\hline Early & $12(13.8)$ & I-III: 162 (79.0) & I-II: 106 (68.8) & I-II: 59 (30.6) & \\
\hline Advanced & $75(86.2)$ & IV: 34 (16.6) & III-IV: 42 (27.3) & III-IV: 131 (67.9) & \\
\hline 0 & - & $136(67.0)$ & - & - & - \\
\hline 1 & - & $35(17.2)$ & $78(50.7)$ & $46(23.8)$ & $42(46.2)$ \\
\hline 4 & $8(9.8)$ & - & $4(2.6)$ & $11(5.7)$ & $8(8.8)$ \\
\hline Khorana score** (bin), n (\%) & $\mathrm{n}=82$ & $\mathrm{n}=203$ & $\mathrm{n}=154$ & $\mathrm{n}=193$ & $\mathrm{n}=91$ \\
\hline$\geq 3$ (high risk) & $45(54.9)$ & $2(1.0)$ & $24(15.6)$ & $63(32.6)$ & $25(27.5)$ \\
\hline$<3$ (low risk) & $37(45.1)$ & $201(99.0)$ & $130(84.4)$ & $130(67.4)$ & $66(72.5)$ \\
\hline
\end{tabular}

VTE: venous thromboembolism; SD: standard deviation; IQR: interquartile range; bin: binary; CRP: C-reactive protein; BMI: body mass index. *Early and advanced stages of cancer for pancreatic patients are defined as curative and non-curative surgery, respectively. ${ }^{* *}$ Khorana score not calculated for patients with abdominal VTE.

groups by Khorana score at diagnosis (excluding those with abdominal VTE). In all groups except for endometrial cancer, a high risk Khorana score was not associated with a higher VTE rate (Fig. 3a-d).

\section{Association with other variables}

The association between the development of VTE in the first year of cancer diagnosis with background and treatment variables were analyzed (Table 3 ). In all cancer groups (excluding cervical cancer where no patient developed a VTE), VTE development was univariately associated with indwelling CVCs $(\mathrm{P}<0.05)$. In endometrial and colorectal cancers, chemotherapy use was significantly associated with VTE development, and in pancreatic, endometrial and ovarian cancers, surgical intervention was significantly associated, even after exclusion of VTE occurring within 90 days of surgery. BMI was only associated with VTE development in ovarian cancer, and full blood count parameters used in the calculation of the Khorana score (platelet count $\geq 350 \times 10^{9}$, Hb level $<100 \mathrm{~g} / \mathrm{L}$ and leukocyte count $>11 \times 10^{9}$ ) were only associated with VTE development in endometrial cancer. Finally, advanced stage of cancer was univariately associated with VTE development in endometrial and ovarian cancer.

Risk factors that remained statistically significant after stepwise logistic regression were indwelling CVC for endometrial, colorectal and ovarian cancers; chemotherapy for pancreatic cancer only; surgery for endometrial and ovarian cancers; $\mathrm{Hb}<100 \mathrm{~g} / \mathrm{L}$ for pancreatic cancer; BMI $>35$ for ovarian cancer; and surgical intervention for endometrial and ovarian cancers.

The VTE rate for patients with indwelling lines were: $34.8 \%(8 / 23)$ for pancreatic; $25.0 \%(2 / 8)$ for endometrial; $22.6 \%(14 / 62)$ for colorectal and $22.7 \%(5 / 22)$ for ovarian. The VTE rate for patients having chemotherapy were: $23.2 \%$ $(13 / 56)$ for pancreatic; $9.1 \%$ (4/44) for endometrial; $16.4 \%$
$(19 / 116)$ for colorectal and $10.1 \%(15 / 148)$ for ovarian. Numbers were too small for further subgroup partitioning within subgroups.

The number of days between cancer and potentially preventable VTE diagnosis was calculated. The median (IQR) time to VTE was 24 days in endometrial cancer ( 7 - 62 days), 61 days $(14-153)$ in ovarian cancer, 90 days $(42-191)$ in pancreatic cancer and 122 days (64 - 153) in colorectal cancer.

\section{Discussion}

This retrospective cohort study of ambulatory cancer patients demonstrates a significant burden of VTE in the first year of diagnosis in four out of five cancer groups analyzed. The exception to this is cervical cancer in which there were no VTE unrelated to an inpatient stay or surgery. However, four out of 91 cervical cancer patients developed VTE which were all associated with short day-case surgical procedures (three EUA and one laparoscopic EUA) defined as "low risk" on local VTE risk assessment and thus did not receive postoperative thromboprophylaxis

The total rates of VTE were $26.8 \%, 5.7 \%, 9.8 \%, 10.2 \%$ and $0.0 \%$ for pancreatic, endometrial, colorectal, ovarian and cervical cancers, respectively. Even excluding those patients who presented with VTE or had thrombosis diagnosed on initial staging scans, the majority of patients with these cancers had a VTE that was potentially preventable with thromboprophylaxis. However, this is not routine clinical practice for outpatients, despite being a quality standard in hospitalized patients [10]. International ACCP guidelines currently recommend that clinicians should not routinely offer pharmacological VTE prophylaxis to outpatients with cancer, but recommend prophylactic LMWH for patients with low bleeding risk and additional risk factors including previous VTE, immobilization, hormonal therapies, angiogenesis inhibitors, thalidomide and lenalidomide [5]. More recent guidelines suggest the 

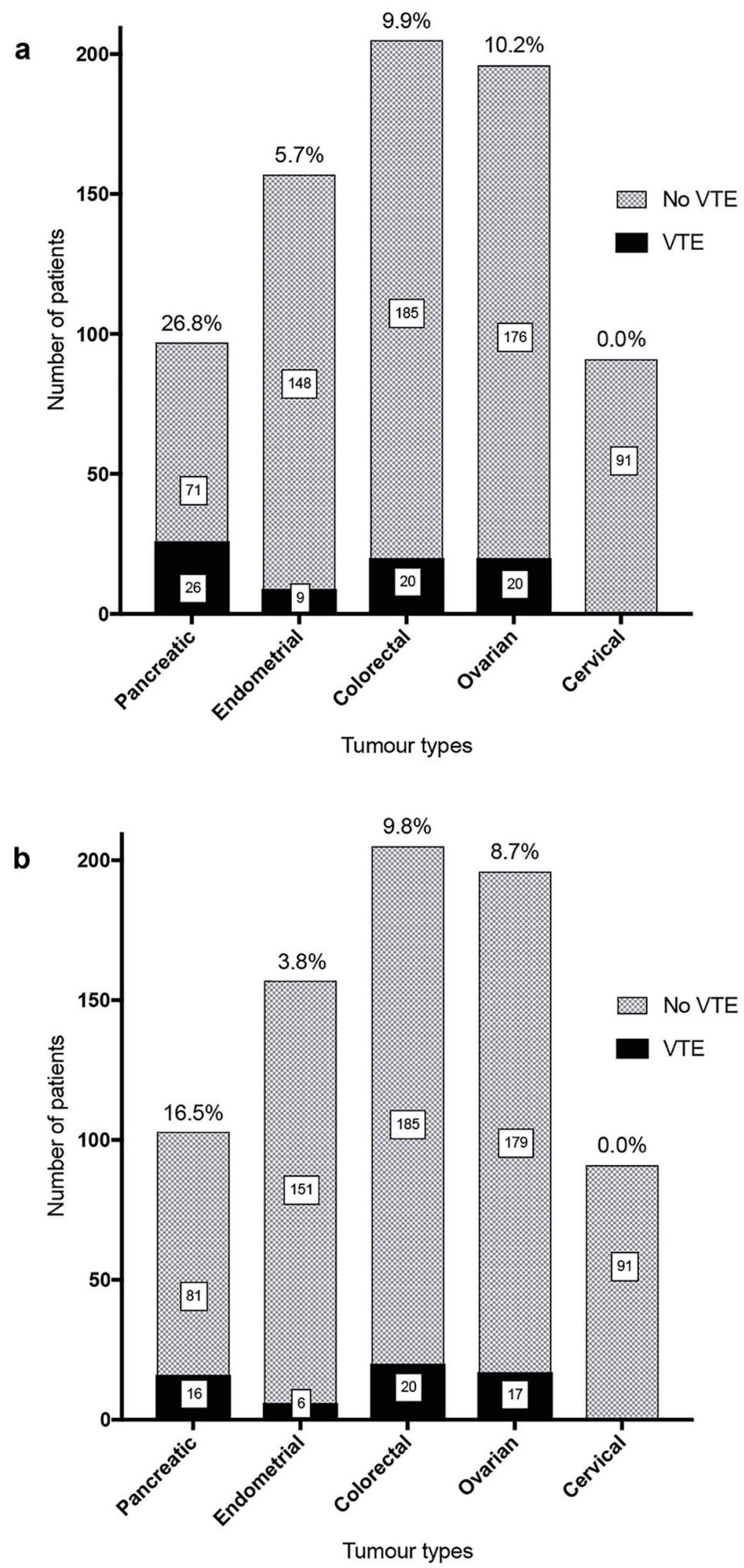

Figure 2. Total VTE (a) and potentially preventable VTE (b) rates for tumor groups. (a) Total VTE rates in the first year of diagnosis were $26.8 \%$ in the pancreatic cancer cohort, $5.7 \%$ in endometrial cancer, $9.8 \%$ in colorectal cancer and $10.2 \%$ in ovarian cancer. No non-hospital associated VTE were identified in the cervical cancer cohort. (b) Excluding VTE present at diagnosis, the rates of VTE in the first year that were potentially preventable with thromboprophylaxis were $16.5 \%$ in pancreatic cancer, $3.8 \%$ in endometrial cancer, $9.8 \%$ in colorectal cancer and $8.7 \%$ in the ovarian cancer cohort. 

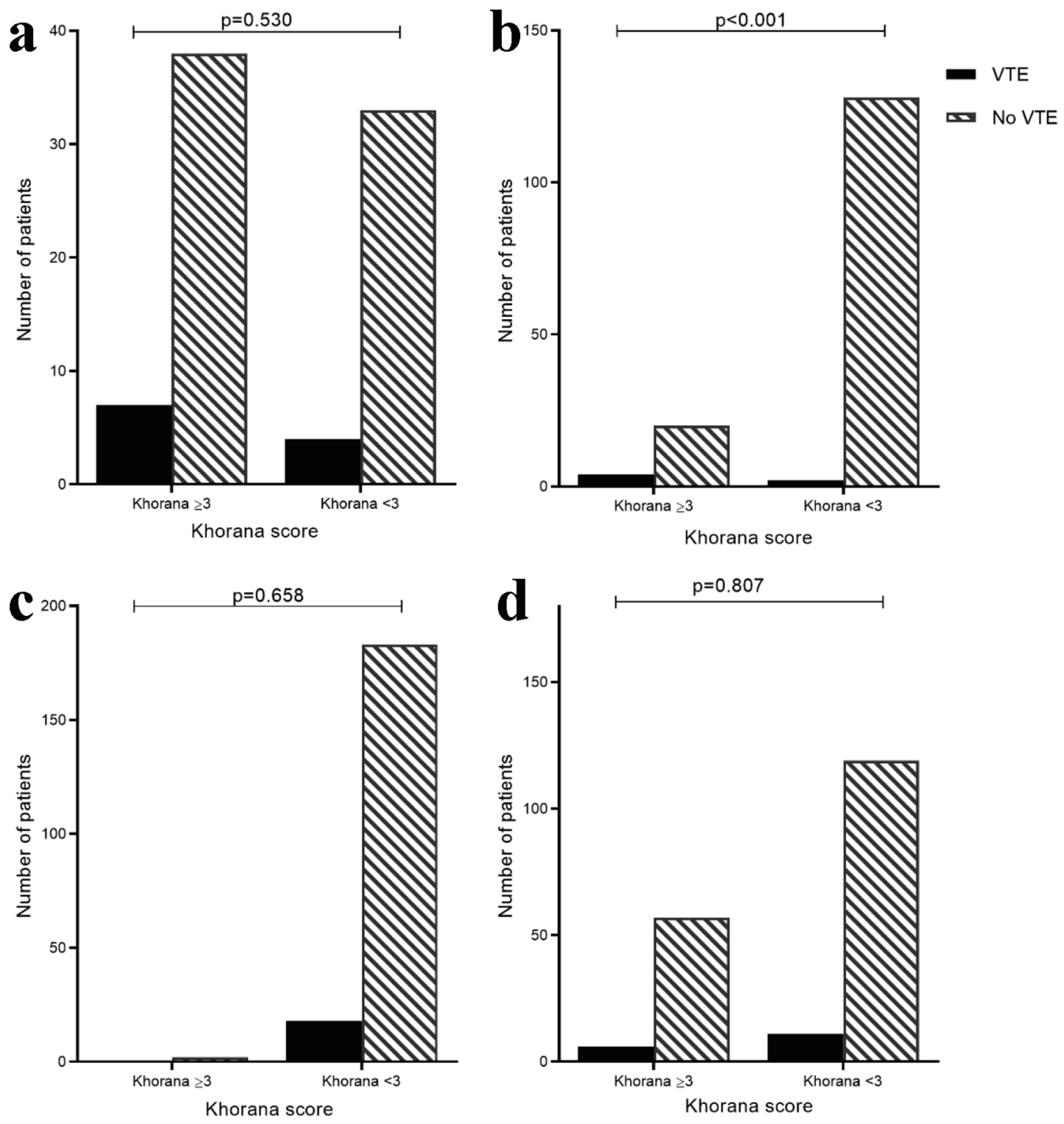

Figure 3. VTE rate by low risk ( $<3$ ) and high risk $(\geq 3)$ Khorana score in pancreatic (a), endometrial (b), colorectal (c) and ovarian (d) cancer patients. Cervical cancer cohort not included as no VTE events. High risk Khorana score predicted VTE in endometrial cancer (VTE rate high-risk Khorana: 4/24 (16.7\%) vs. low-risk: 2/130 (1.5\%); P < 0.001) but not in other cancer groups.

use of the Khorana score for risk stratification [8].

However, our data suggest that the Khorana score is not valuable in risk stratifying patients with pancreatic, colorectal and ovarian cancers, although a higher Khorana score was predictive of 1-year VTE development in endometrial cancer. Furthermore, use of the Khorana score to define who may benefit from prophylactic anticoagulation in the cervical cancer cohort would have potentially meant all 25 patients with a high-risk score were unnecessarily anticoagulated, since none developed a VTE which was not associated with an inpatient admission or surgery.

We investigated other variables associated with VTE development in ambulatory cancer patients. Risk factors which remained statistically significant after stepwise logistic regression were indwelling CVC for endometrial, colorectal and ovarian cancers; chemotherapy for pancreatic cancer only; surgery for endometrial and ovarian cancers; $\mathrm{Hb}<100 \mathrm{~g} / \mathrm{L}$ for pancreatic cancer and BMI $>35$ for ovarian cancer. The VTE rate for patients with indwelling lines was particularly high, with around a quarter of endometrial, colorectal and ovarian; and over a third of pancreatic cancer patients with CVC developing VTE, either local or systemic.

Improving risk stratification in ambulatory cancer patients is likely to have an impact on morbidity due to the high burden of VTE incidence demonstrated in this study. The VTE risk in patients with malignancy is greatest in the first year follow- 


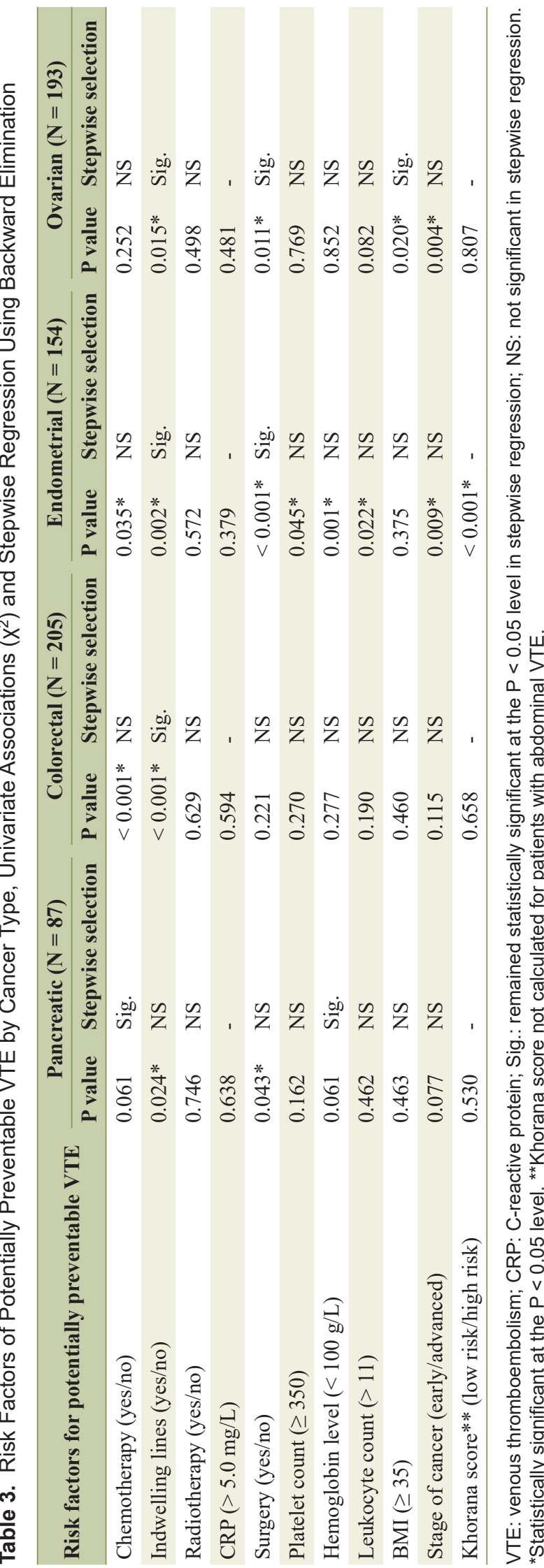

ing diagnosis, so it is important to develop simple and reliable methods to risk stratify in the MDT or outpatient department soon after diagnosis [11]. Despite some studies suggesting that bleeding rates with use of anticoagulants in cancer patients are higher than in patients without malignancy, large studies have refuted this and there is no evidence suggesting this is the case with primary thromboprophylaxis $[7,12]$.

An obvious limitation is that this is a retrospective study. However, it includes a large cohort and provides important information to guide future prospective investigations of thromboprophylaxis in ambulatory cancer patients, in particular, factors to identify those at high risk. Oncology care was delivered at our tertiary center but some patients may have consulted their local hospitals with acute presentations, including of VTE. Thus, our data may in fact underestimate the incidence of VTEs and this may explain in part why there were no VTE diagnoses in the cervical cancer cohort. Future multicenter prospective studies looking at additional tumor groups would be of value in further evaluating the extent of the clinical problem.

In summary, this retrospective cohort study at a tertiary oncology center has identified a significant burden of VTE in pancreatic, endometrial, colorectal and ovarian cancer cohorts. Khorana score did not predict risk of VTE development in pancreatic, colorectal, ovarian or cervical cancer groups, although it was predictive in patients with endometrial cancer. The major variable associated with VTE development was indwelling CVC with VTE rates of 23-35\% depending on tumor type. Our data suggest a role for clinical trials of routine thromboprophylaxis in targeted groups of cancer outpatients, such as those with pancreatic cancer, or with endometrial, colorectal and ovarian cancers and indwelling CVCs.

\section{Author Contributions}

Dr Jessica George (joint first author) performed research including data collection, and wrote the paper. Dr Keziah Austin (joint first author) performed research including data collection, and wrote the paper. Miss Emily Robinson statistically analyzed the data, created tables and wrote the "statistical analysis" section of the paper. Dr Marie Scully designed research, study and provided expert clinical knowledge to revise critically. Dr Mari Thomas designed research, study and provided expert clinical knowledge to revise critically.

\section{Competing Interests}

The authors have no competing interests.

\section{References}

1. Blom JW, Doggen CJ, Osanto S, Rosendaal FR. Malignancies, prothrombotic mutations, and the risk of venous thrombosis. JAMA. 2005;293(6):715-722.

2. Khorana AA. Venous thromboembolism and prognosis in 
cancer. Thromb Res. 2010;125(6):490-493.

3. Hutten BA, Prins MH, Gent M, Ginsberg J, Tijssen JG, Buller HR. Incidence of recurrent thromboembolic and bleeding complications among patients with venous thromboembolism in relation to both malignancy and achieved international normalized ratio: a retrospective analysis. J Clin Oncol. 2000;18(17):3078-3083.

4. Prandoni P, Lensing AW, Piccioli A, Bernardi E, Simioni P, Girolami B, Marchiori A, et al. Recurrent venous thromboembolism and bleeding complications during anticoagulant treatment in patients with cancer and venous thrombosis. Blood. 2002;100(10):3484-3488.

5. Kearon C, Akl EA, Comerota AJ, Prandoni P, Bounameaux H, Goldhaber SZ, Nelson ME, et al. Antithrombotic therapy for VTE disease: Antithrombotic Therapy and Prevention of Thrombosis, 9th ed: American College of Chest Physicians Evidence-Based Clinical Practice Guidelines. Chest. 2012;141(2 Suppl):e419S-e496S.

6. Barbar S, Noventa F, Rossetto V, Ferrari A, Brandolin B, Perlati M, De Bon E, et al. A risk assessment model for the identification of hospitalized medical patients at risk for venous thromboembolism: the Padua Prediction Score. J Thromb Haemost. 2010;8(11):2450-2457.

7. Di Nisio M, Porreca E, Otten HM, Rutjes AW. Primary prophylaxis for venous thromboembolism in ambulatory cancer patients receiving chemotherapy. Cochrane Database Syst Rev. 2014;8:CD008500.

8. Watson HG, Keeling DM, Laffan M, Tait RC, Makris M, British Committee for Standards in H. Guideline on aspects of cancer-related venous thrombosis. Br J Haematol. 2015;170(5):640-648.

9. Khorana AA, Kuderer NM, Culakova E, Lyman GH, Francis CW. Development and validation of a predictive model for chemotherapy-associated thrombosis. Blood. 2008;111(10):4902-4907.

10. High Quality VTE Prevention. Guidance for Commissioners. NHS England, May 2013. https://www.england. nhs.uk/wp-content/uploads/2013/08/vte-prev-guidemay2013-22.7.13.pdf.

11. Chew HK, Wun T, Harvey D, Zhou H, White RH. Incidence of venous thromboembolism and its effect on survival among patients with common cancers. Arch Intern Med. 2006;166(4):458-464.

12. Kuderer NM, Khorana AA, Lyman GH, Francis CW. A meta-analysis and systematic review of the efficacy and safety of anticoagulants as cancer treatment: impact on survival and bleeding complications. Cancer. 2007;110(5):1149-1161. 